\title{
Clinical features, muscle biopsy and molecular genetics
}

\author{
Paulo José Lorenzoni', Rosana H. Scola', Cláudia S. Kamoi Kay', Raquel C. Arndt', \\ Aline A. Freund', Isac Bruck', Mara Lúcia S.F. Santos ${ }^{3}$, Lineu C. Werneck'
}

\begin{abstract}
Objective: The aim of the study was to analyze a series of Brazilian patients suffering from MELAS. Method: Ten patients with MELAS were studied with correlation between clinical findings, laboratorial data, electrophysiology, histochemical and molecular features. Results: Blood lactate was increased in eight patients. Brain image studies revealed a stroke-like pattern in all patients. Muscle biopsy showed ralled-red fibers (RRF) in $90 \%$ of patients on modified Gomori-trichrome and in $100 \%$ on succinate dehydrogenase stains. Cytochrome c oxidase stain analysis indicated deficient activity in one patient and subsarcolemmal accumulation in seven patients. Strongly succinate dehydrogenase-reactive blood vessels (SSV) occurred in six patients. The molecular analysis of tRNA ${ }^{\text {Leu(UUR) }}$ gene by PCR/RLFP and direct sequencing showed the A3243G mutation on mtDNA in 4 patients. Conclusion: The muscle biopsy often confirmed the MELAS diagnosis by presence of RRF and SSV. Molecular analysis of tRNA ${ }^{\text {Leu(UUR) }}$ gene should not be the only diagnostic criteria for MELAS.
\end{abstract}

KEY WORDS: MELAS, mitochondrial myopathies, biopsy, mitochondrial DNA, genetics.

\section{MELAS: manifestações clínicas, biópsia muscular e estudo molecular}

Resumo - Objetivo: O objetivo deste estudo foi analisar uma série de pacientes brasileiros portadores de MELAS. Método: Dez pacientes com MELAS foram estudados com correlação entre manifestações clínicas, alterações laboratoriais, estudo eletrofisiológico, histoquímico e molecular. Resultados: $O$ nível de lactato sérico estava aumentado em 8 pacientes. $O$ estudo das imagens do crânio revelou padrão semelhante ao de AVC isquêmico em todos os pacientes. A biópsia muscular mostrou fibras rajadas vermelhas (RRF) em $90 \%$ dos pacientes na coloração pelo tricrômio de Gomori modificado e em $100 \%$ na reação histoquímica pela desidrogenase succicínica (SDH). A análise da coloração pela citocromo c oxidase indicou atividade deficiente em um paciente e acúmulo subsarcolemal em sete pacientes. Vasos com forte reação para SDH (SSV) ocorreram em seis pacientes. $O$ estudo molecular do gene tRNA ${ }^{\text {Leu(UUR) }}$ por PCR/RLFP e seqüenciamento direto mostrou a mutação A3243G no DNAmt de 4 pacientes. Conclusão: A biópsia muscular frequentemente confirma o diagnóstico de MELAS pela presença de RRF e SSV. O estudo molecular do gene tRNA ${ }^{\text {Leu(UUR) }}$ não deve ser o único critério diagnóstico para MELAS.

PALAVRAS-CHAVE: MELAS, miopatias mitocondriais, biópsia, DNA mitocondrial, genética.

Mitochondrial encephalomyopathies are a heterogeneous group of clinical syndromes, with various biochemical defects of the respiratory chain due to primary defects in cellular mitochondria ${ }^{1-5}$. Mitochondrial myopathy, encephalopathy, lactic acidosis, and stroke-like episodes (MELAS) is one of occurring mitochondrial disease which includes in its definition encephalopathy, frequently with seizures and progressive dementia; stroke-like episodes at a young age; and biochemical evidence for mitochondrial defects, such as lactic acidosis or ragged-red fibers (RRF) in the muscle biopsy $y^{3,4,6}$.

The pathogenesis of stroke-like episodes in MELAS is controversial; the two major hypotheses posit dependence on metabolic dysfunction or angiopathy in

\footnotetext{
${ }^{1}$ Neurology/Neuromuscular Division, Internal Medicine Department, Universidade Federal do Paraná, Curitiba PR, Brazil; ${ }^{2}$ Neuropediatric Division, Pediatric Department, Universidade Federal do Paraná, Curitiba PR, Brazil; ${ }^{3}$ Pediatric Neurology Department, Hospital Pequeno Príncipe, Curitiba PR, Brazil. This study was supported by Fundação Araucária, CAPES and CNPq.
}

Received 5 December 2008, received in final form 30 March 2009. Accepted 28 May 2009.

Dra. Rosana Herminia Scola - Serviço de Doenças Neuromusculares / Hospital de Clínicas da UFPR - Rua General Carneiro 181 / $3^{\circ}$ andar - $80060-900$ Curitiba PR - Brasil.E-mail: scola@hc.ufpr.br 
the brain, but these two elements are thought to be related ${ }^{7}$.

Since its first description, few patients with this disease have been identified in $\mathrm{Brazi}^{8-10}$. In this study we analyze the clinical and laboratory manifestations, brain images, histological and molecular findings in 10 Brazilian patients suffering from MELAS.

\section{METHOD}

A retrospective analysis of 4400 muscle biopsies performed from January 1978 to January 2008 disclosed ten patients with a diagnosis of MELAS, according to the following requisite clinical features: (1) at least one of the following: seizures, dementia, recurrent headache or vomiting; (2) stroke-like episodes at a young age (with computerized tomographic or magnetic resonance imaging evidence of focal brain abnormalities); and (3) lactic acidosis, RRF, or both. Relevant data, including clinical evaluation, age, gender, course of the disease, serum muscle enzyme levels, blood and cerebrospinal fluid (CSF) lactate levels, muscle cytochrome c oxidase (COX) activity, electrocardiogram (ECG), needle electromyography (EMG), nerve conduction study, brain images and histological/histochemical aspects of the each muscle biopsy, were collected. The consent for muscle biopsy and mitochondrial DNA (mtDNA) tests was obtained in the out-patient clinic or during hospital admission for diagnostic investigation. All studies were performed following the acquisition of informed consent.

\section{Clinical evaluation}

The time of progression of the disease was considered as the interval between the stroke-like episodes and the definitive diagnosis by muscle biopsy. Other relevant data, including clinical evaluation, age, gender, course of disease, and family history, were collected.

\section{Laboratorial analysis}

Abnormal serum levels of creatine kinase (CK), aldolase (AL), blood and CSF lactate were registered as a proportion reflecting their increase above normal limits. COX activity was measured in muscle biopsy extracts.

\section{Electrophysiological findings}

The EMG pattern was classified as normal, myopathic, denervated or mixed (myopathic with denervation findings), according to standard procedures. Motor and sensory nerve conduction studies were performed. The ECG abnormalities were reported.

\section{Brain images}

The radiologic findings (computed tomographic (CT) or magnetic resonance imaging (MRI)) were separated by lesion location and presence of basal ganglia calcifications.

\section{Muscle biopsy analysis}

Muscle biopsies were frozen in liquid nitrogen and cryostat sections were stained histologically; histochemical reactions were performed from the routine examination of the reaction, according to standard procedures ${ }^{11}$. The frequency of deficient muscle fibers on COX stain; and of RRF on modified Gomoritrichrome (MGT) and succinate dehydrogenase (SDH) stains were determined by counting in approximately 500 to 1000 muscle fibers of each specimen. In all specimens, the blood vessels (intramuscular arteries) were identified comparing the hematoxylin-eosin, MGT and SDH stains. The blood vessel characteristics identified by SDH staining were categorized as follows: unstained or faintly stained vessels walls (normal); or strongly succinate dehydrogenase-reactive blood vessels (SSV) or darkly stained vessels walls with dense granules (abnormal).

\section{Molecular analysis}

The mtDNA was isolated from skeletal muscle from the quadriceps or biceps brachii using a modified phenol/chloroform method. The tRNA ${ }^{\text {Leu(UUR) }}$ gene of mtDNA was analyzed by a combination of polymerase chain reaction with restriction fragment length polymorphism (PCR/RFLP) and direct sequencing.

In the first PCR/RFLP, used to detect a point mutation, an A to $G$ transition at nucleotide 3243 (A3243G), we used oligonucleotide primers corresponding to positions nt 3116-3134 (forward) and nt 3353-3333 (backward) to amplify the mtDNA putative mutation by conventional polymerase chain reaction (PCR) with Taq DNA polymerase. In the second PCR/RLFP, used to detect a point mutation, a $\mathrm{T}$ to $\mathrm{C}$ transition at nucleotide 3271 (T3271C), we used oligonucleotide primers corresponding to positions nt 3148-3169 (forward) and nt 3295-3272 (backward) for conventional PCR amplification with Taq DNA polymerase.

The PCR conditions were as follows: 30 cycles of denaturation $\left(94^{\circ} \mathrm{C}\right.$ for $\left.1 \mathrm{~min}\right)$, annealing $\left(55^{\circ} \mathrm{C}\right.$ for $1 \mathrm{~min}$ ) and primer extension $\left(72^{\circ} \mathrm{C}\right.$ for $\left.1 \mathrm{~min}\right)$. The first PCR produced a 238-base-pair (bp) fragment which was digested for 16 hours at $37^{\circ} \mathrm{C}$ with the restriction endonuclease Hae III. The second PCR produced a 148 bp fragment which was digested for 6 hours at $37^{\circ} \mathrm{C}$ with the restriction endonuclease $D$ de I.

These products from PCR/RFLP were analyzed on a 12\% nondenaturing polyacrylamide gel (run at $200 \mathrm{~V}$ for 2 hours) and the bands were visualized by standard silver staining. In the first PCR/RLFP, the pattern observed with normal mtDNA consist of three bands (169, 37 and $32 \mathrm{bp}$ ), whereas, the presence of the A3243G mutation creates an additional Hae III restriction site and the 169 bp fragment is cleaved into two fragments of 97 and 72 bp. In the second PCR/RLFP, the pattern observed with normal mtDNA consists of two bands (103 and 45 bp), whereas the presence of the T3271C mutation creates an additional Dde I restriction site and the $103 \mathrm{bp}$ fragment is cleaved into two fragments of 79 and $24 \mathrm{bp}$.

If any mutation was observed by both PCR/RLFP, the tRNA ${ }^{\text {Leu(UUR) }}$ gene was submitted to direct sequencing.

For direct sequencing we used the nt 3116-3134 and nt 33533333 oligonucleotide primers to amplify the tRNA ${ }^{\text {Leu(UUR) }}$ gene by 
conventional PCR with Taq DNA polymerase. The first PCR conditions were 30 cycles of denaturation $\left(94^{\circ} \mathrm{C}\right.$ for $\left.1 \mathrm{~min}\right)$, annealing $\left(55^{\circ} \mathrm{C}\right.$ for $\left.1 \mathrm{~min}\right)$ and primer extension $\left(72^{\circ} \mathrm{C}\right.$ for $\left.1 \mathrm{~min}\right)$; followed by purification of the PCR fragment by enzymatic method. These purified PCR fragments were submitted to a second PCR using Big Dye Mix (Applied Biosystems). The second PCR conditions were 35 cycles of denaturation $\left(96^{\circ} \mathrm{C}\right.$ for $\left.15 \mathrm{sec}\right)$, annealing $\left(50^{\circ} \mathrm{C}\right.$ for $15 \mathrm{sec}$ ) and primer extension $\left(60^{\circ} \mathrm{C}\right.$ for $\left.4 \mathrm{~min}\right)$; followed by PCR fragment purification by the isopropanolol method. These amplified fragments were directly sequenced in forward direction on ABI PRISM 3100 Avant Genetic Analyzer and sequences were compared to the revised Cambridge reference sequence ${ }^{12}$.

\section{RESULTS}

The sample consisted of 10 patients ( 4 female and 6 male), aged 3 to 27 years, with a predominance of child patients in this series (in 6 patients onset was before age 15 years). The early development was normal in 9 of 10 patients (90\%). The time of the progression of the disease varied to a maximum of 90 months, with a mean time of 27.5 months. The families had no history of possible mitochondrial disorders (Table 1).

The stroke-like episodes were present in all cases. The other symptoms reported were vomiting (10/10), headache $(8 / 10)$, seizures $(6 / 10)$, weakness $(6 / 10)$, dementia $(5 / 10)$, hearing loss $(3 / 10)$, short stature $(2 / 10)$, ocular symptoms $(3 / 10)$, ataxia (1/10) and facial neuropathy (1/10) (Table 1).

Blood lactate levels varied from one to three times normal levels in eight patients. CSF lactate level doubled in one patient during the stroke-like episode (Table 1).

Serum CK levels varied from normal (6 patients) to twice normal ( 2 patients). Serum AL levels were normal in all patients (Table 1).

Analysis of COX activity from muscle extract was possible in 4 cases, of which two indicated COX deficiency (Table 1).

The EMG pattern, observed in five patients, was myopathic in two, neurogenic (only in facial muscles) in one and normal in two. Nerve conduction studies showed facial neuropathy in one patient (case 5), but were normal in the other patients.

ECG, performed in 6 patients, was normal in three but revealed Wolf-Parkinson-White syndrome in one, right bundle branch conduction disturbance in one and left ventricular hypertrophy together with nonspecific $\mathrm{T}$ wave abnormality in another.

Table 1. Clinical and laboratorial description of MELAS patients.

\begin{tabular}{|c|c|c|c|c|c|c|c|c|c|c|}
\hline Case & 1 & 2 & 3 & 4 & 5 & 6 & 7 & 8 & 9 & 10 \\
\hline Age (years) & 12 & 22 & 9 & 3 & 4 & 15 & 14 & 8 & 25 & 27 \\
\hline Gender & M & $\mathrm{F}$ & M & M & $\mathrm{F}$ & $\mathrm{F}$ & M & M & $\mathrm{F}$ & M \\
\hline Evolution time (months) & 36 & 6 & 90 & 12 & 10 & 1 & 48 & 12 & 33 & 0 \\
\hline Family history & - & - & - & - & - & - & - & - & - & - \\
\hline Developmental delay & - & - & - & + & - & - & - & - & - & - \\
\hline Stroke_like episodes & + & + & + & + & + & + & + & + & + & + \\
\hline Vomiting & + & + & + & + & + & + & + & + & + & + \\
\hline Headache & + & + & + & + & - & + & + & + & - & + \\
\hline Seizures & + & + & + & - & - & + & + & + & - & - \\
\hline Weakness & + & - & - & - & - & + & + & + & + & + \\
\hline Dementia & - & - & - & - & - & + & + & + & + & + \\
\hline Hearing loss & - & - & + & - & - & - & + & - & - & + \\
\hline Short stature & - & - & - & - & - & - & + & - & - & + \\
\hline Ocular symptons & - & - & - & - & - & - & + & - & + & + \\
\hline Ataxia & - & - & + & - & - & - & - & - & - & - \\
\hline Facial neuropathy & - & - & - & - & + & - & - & - & - & - \\
\hline Increased blood lactate & + & ND & + & + & + & + & + & + & _ & + \\
\hline Increased CSF lactate & ND & ND & ND & ND & ND & ND & + & - & ND & ND \\
\hline Increased CK & - & ND & - & - & - & - & + & - & ND & + \\
\hline Increased AL & - & ND & ND & ND & - & - & - & - & ND & ND \\
\hline COX deficiency* & + & + & ND & _ & _ & ND & ND & ND & ND & ND \\
\hline
\end{tabular}

F: female; M: male; CSF: cerebrospinal fluid; CK: creatine kinase; AL: aldolase; COX: cytocrome c oxidase; *COX deficient activity in muscle extract; RRF: ragged_red fiber; MGT: modified Gomori trichome; SDH: succinate dehydrogenase; +: presented; _: absent; ND: not done. 
Table 2. Imaging, histological and molecular characteristics of MELAS patients.

\begin{tabular}{|c|c|c|c|c|c|c|c|c|}
\hline \multirow[b]{2}{*}{ Case } & \multirow[b]{2}{*}{ Brain abnormality localization } & \multicolumn{3}{|c|}{ Muscle biopsy (\%) } & \multirow[b]{2}{*}{ SSV $\%$} & \multicolumn{3}{|c|}{ tRNALeu(UUR) gene mutation } \\
\hline & & *MGT & ${ }^{*} \mathrm{SDH}$ & cox- & & A3243G\# & T3271C\# & Others\#\# \\
\hline 1 & $\mathrm{CT}$ : bilateral temporal-occipital & 10.0 & 11.8 & ND & 33.3 & - & - & - \\
\hline 2 & $\mathrm{CT}$ : right frontal-temporal & 0.5 & 2.1 & ND & 0 & ND & ND & ND \\
\hline 3 & MRI: bilateral basal ganglia & 2.4 & 5.3 & 0 & 0 & ND & ND & ND \\
\hline 4 & CT: left temporal-occipital & 0 & 0.7 & 0 & 0 & - & - & - \\
\hline 5 & MRI: bilateral occipital & 6.3 & 7.5 & 0 & 0 & ND & ND & ND \\
\hline 6 & $\mathrm{CT}$ : left temporal-parietal-occipital & 0.4 & 3.8 & 0 & 37.5 & + & ND & ND \\
\hline 7 & MRI: left temporal-parietal-occipital & 2.2 & 5.4 & 0 & 70 & + & ND & ND \\
\hline 8 & MRI: bilateral frontal-parietal-temporal & 6.9 & 9.2 & 0 & 75 & + & ND & ND \\
\hline 9 & MRI: right cerebelar & 0.8 & 2.0 & 32.7 & 38 & - & - & - \\
\hline 10 & MRI: left basal ganglia & 6.7 & 11.4 & 0 & 14.2 & + & ND & ND \\
\hline
\end{tabular}

SSV: strongly succinate dehydrogenase-reactive blood vessels; *MGT: ragged-red fiber stained by modified Gomori trichome; *SDH: ragged-red fiber stained by succinate dehydrogenase; COX-: cytocrome c oxidase deficient on muscle fiber stain; CT: computed tomography; MRI: magnetic resonance imaging; "molecular analysis by PCR/RLFP; "\#molecular analysis of others mutation in tRNALeu(UUR) gene by direct sequencing; +: present; -: absent; ND: not done.
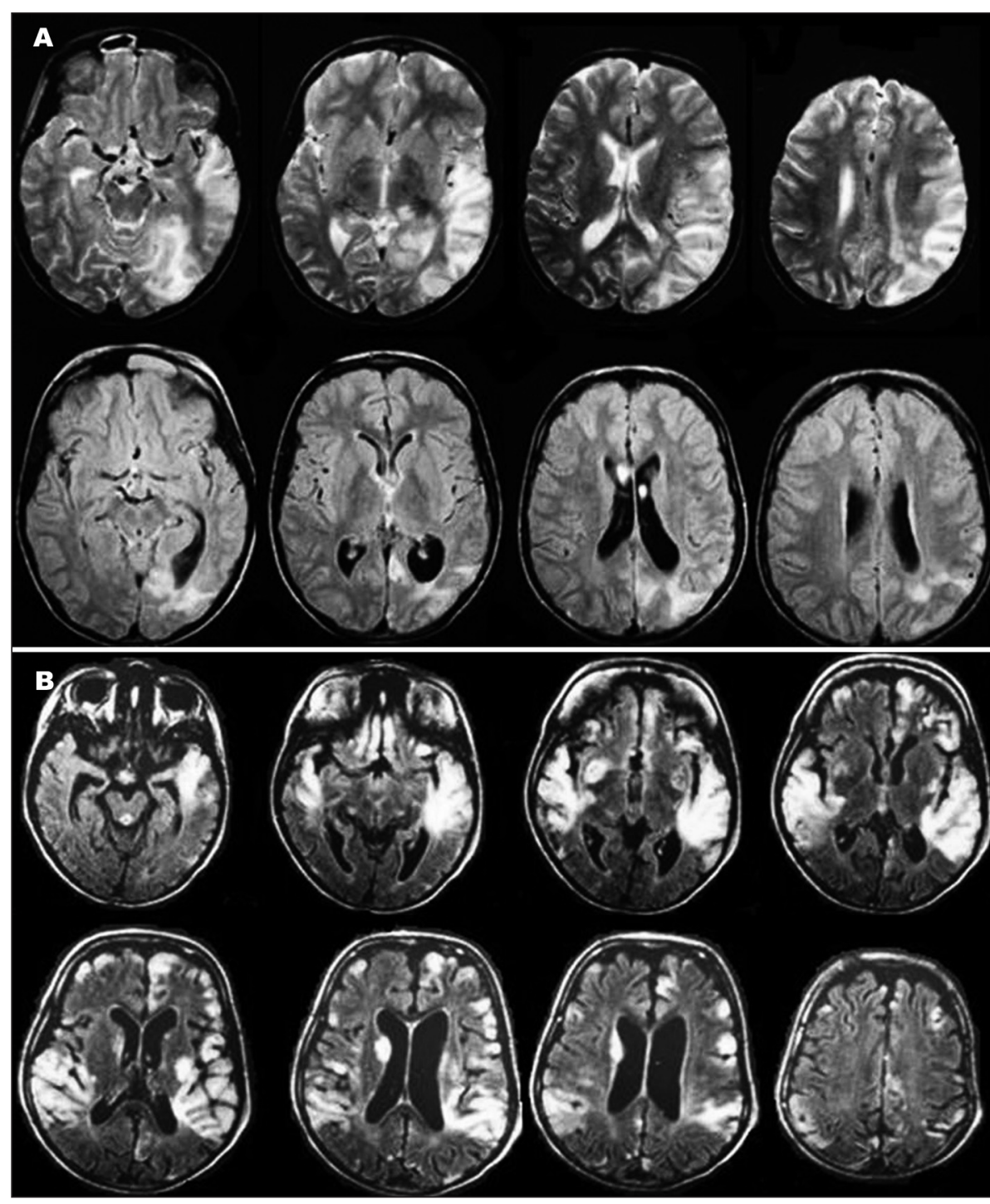

Fig 1. Brain magnetic resonance imaging confirms a unilateral stroke-like lesion pattern in case 7 in axial T2 and FLAIR weighted images [A], and bilateral in case 8 in axial FLAIR weighted images [B]. 
Brain image study, obtained from computed tomography in four patients and in six by magnetic resonance imaging, showed a stroke-like pattern in all patients. The lesion was unilateral in six patients and bilateral in four (Fig 1). The most common lesion locations were temporal-occipital, frontal-temporal, basal ganglia, temporal, occipital, temporal-parietal-occipital, frontal-parietal-temporal and cerebellar. The $\mathrm{CT}$ revealed basal ganglia calcifications in two patients (Table 2).

The MGT and SDH stain were performed in all cases, but COX staining was performed only in 8 cases. The RRF occurred in nine cases by MGT stain and in all cases by SDH stain, but the proportion of RRF ranged from $0.4 \%$ to $11.6 \%$ on the MGT stain and from $0.7 \%$ to $11.8 \%$ on the SDH stain. Greater than 2\% RRF was found in six of nine patients with the MGT stain and in eight cases with the SDH stain. COX deficient activity was found only in the muscle fibers of case 9 , but COX subsarcolemmal accumulation occurred in 7 out 8 patients (Table 2 and Fig 2).

SSV occurred in six cases and its frequency ranged from $14.2 \%$ to $75 \%$ in these cases (Table 2 and Fig 3).

The molecular analysis was possible in the muscle biopsy from 7 patients (Table 2). The A3243G mutation was detected by PCR/RLFP in four (Fig 4A). Others mtDNA mutations were not found in tRNA $A^{\text {Leu(UUR) }}$ gene by PCR/ RLFP to T3271C or direct sequence analysis (Fig 4B and 4C).
In the patients with MELAS related to the A3243G mutation, the most common findings were a mean time of progression of 15.2 months, normal levels of serum CK (two cases), normal levels of serum AL (three cases), EMG with myopathic pattern (two cases), normal COX activity (all cases) and SSV (all cases).

\section{DISCUSSION}

Early disease development is normal in about $90 \%$ of MELAS patients ${ }^{3,4,13}$. The onset of this disease occurs before age 40 and is often seen in childhood, with gradual deterioration ${ }^{3,4,14}$. Clinical symptoms are highly variable across patients ${ }^{2}$. The most common feature in MELAS is episodic headaches with vomiting; this symptom was present in almost all patients ${ }^{3,4,15}$. Seizures occur in the vast majority of patients $(95 \%)^{3,4,15}$. The other symptoms were not sufficiently severe to bring the patients to medical attention. Thus, the stroke-like episodes may appear abruptly in otherwise normal-appearing children ${ }^{4,15}$.

Having an affected family member was a factor in $23 \%$ of patients with MELAS ${ }^{4,13}$. However, $86 \%$ of the oligosymptomatic and asymptomatic relatives of MELAS patients are identified only by muscle biopsy or molecular studies ${ }^{4,3}$. In the absence of a member of the family with the full syndrome, and in the absence of molecular genetic studies, oligosymptomatic patients with MELAS are dif-

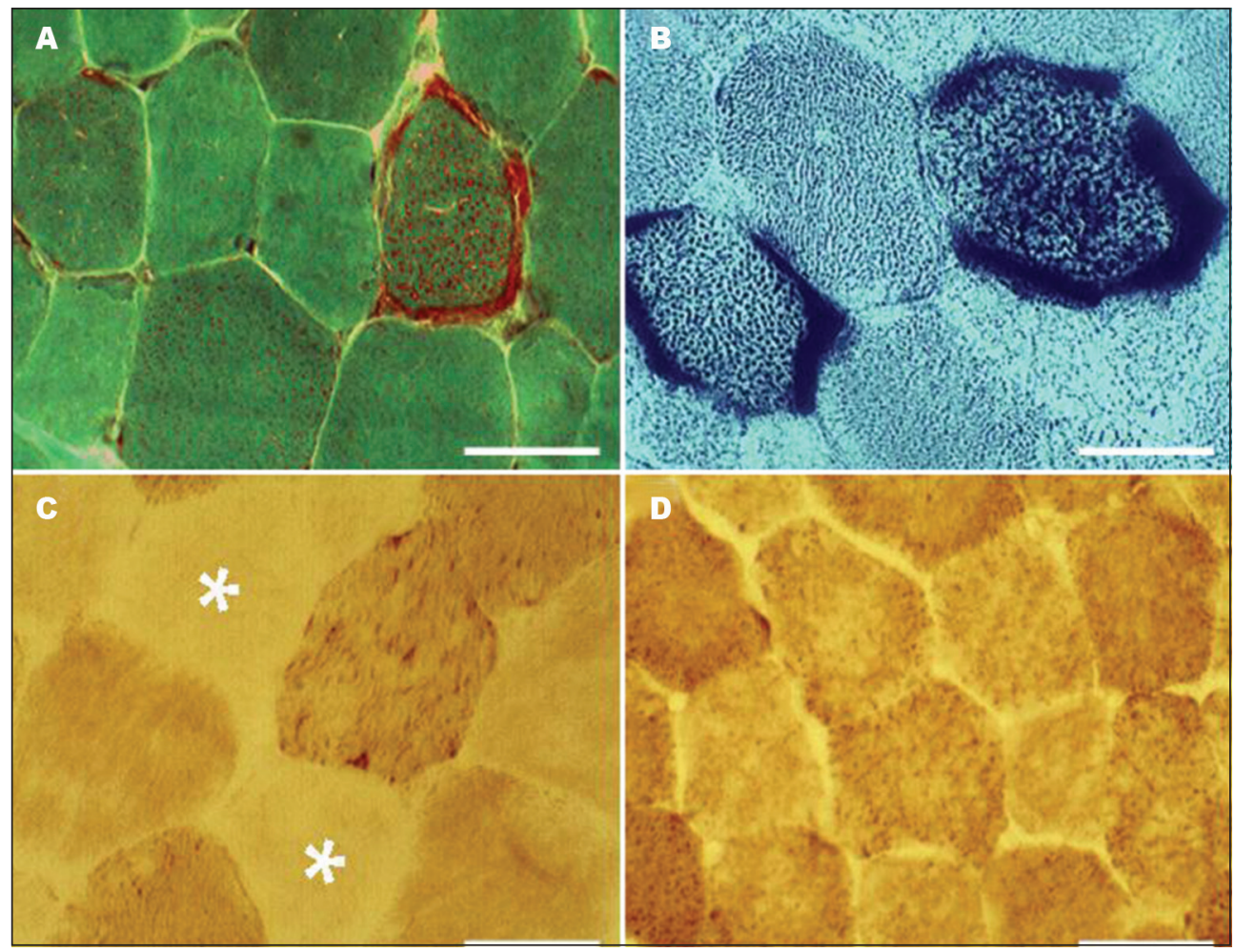

Fig 2. Muscle biopsy: [A] ragged-red fibers on modified Gomori-trichrome stain (case 8); [B] ragged-red fibers on succinate dehydrogenase stain (case 8); [C] deficient muscle fiber activity (*) on cytochrome c oxidase stain (case 9); and [D] normal muscle fiber activity on cytochrome c oxidase stain (case 8). Bar=50um. 


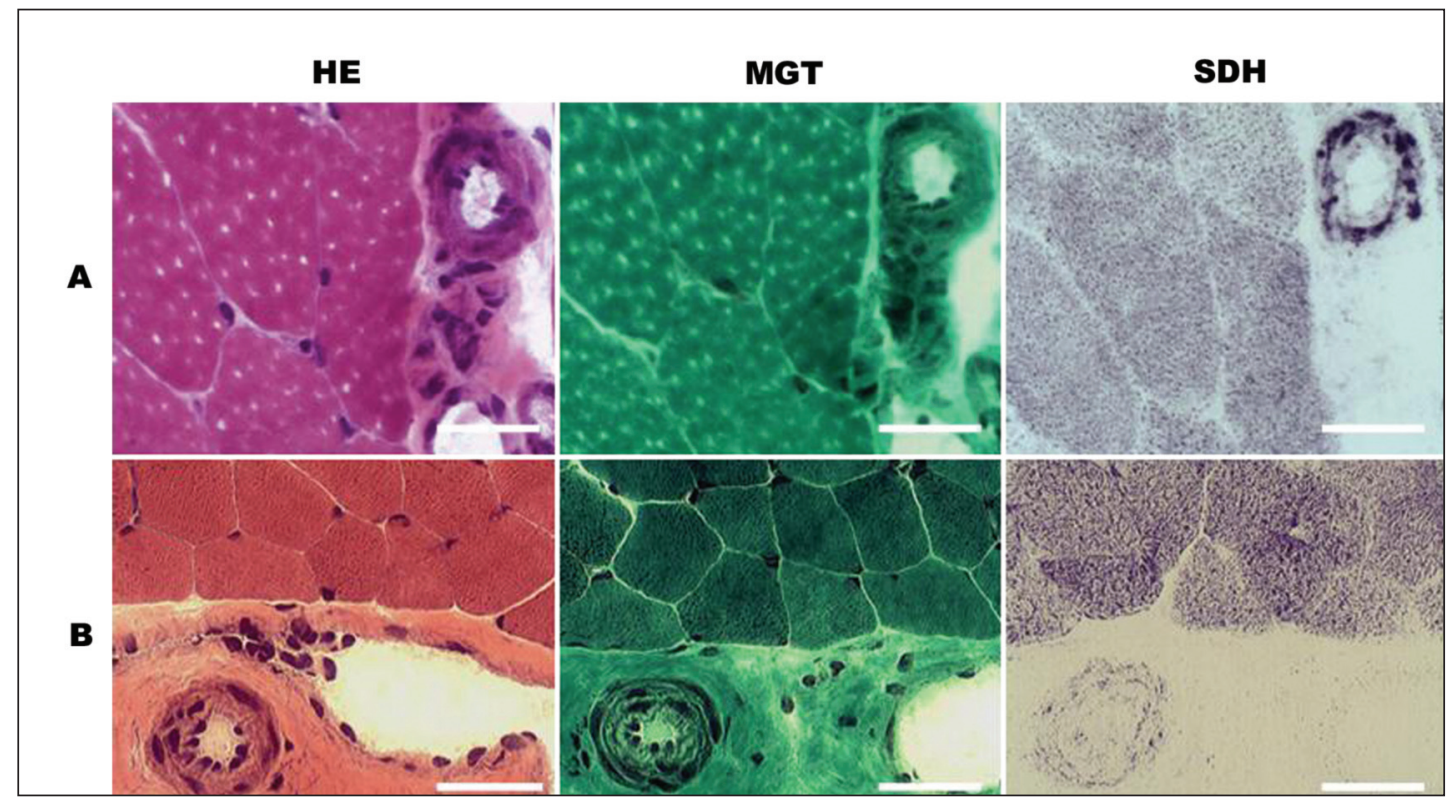

Fig 3. Muscle biopsy: succinate dehydrogenase (SDH) stain of muscle biopsy showing SSV in case 6 [A] and normal vessels walls in case $5[B]$ compared with hematoxylin-eosin (HE) and modified Gomori trichome (MGT) stains. Bar=50um.

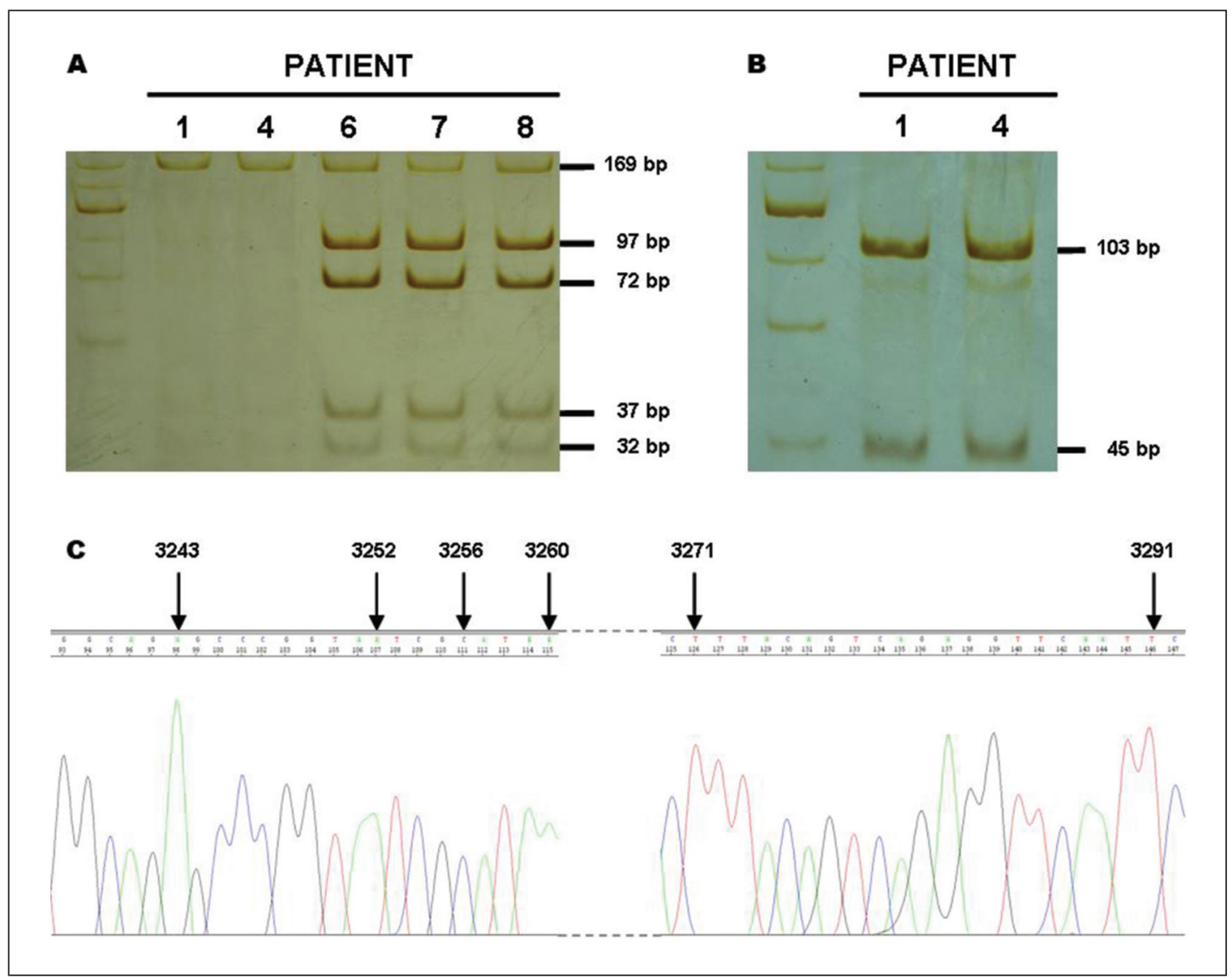

Fig 4. Molecular analysis of mtDNA: [A] PCR/RFLP analysis for A3243G mutation showed normal mtDNA with fragments of 169, 37 and 32 bp, whereas mutant mtDNA has fragments of 169, 97, 72, 37 and 32 bp; [B] PCR/RFLP analysis for T3271C showed normal mtDNA pattern observed by the presence of 103 and 45 bp fragments; and [C] mtDNA sequencing showing normal tRNA ${ }^{\text {Leu(UUR) }}$ in case 4 (point mutation for MELAS in this gene are highlighted by arrows). 
ficult to classify ${ }^{13}$. The family history was not consistent with maternal inheritance and none of our patients' family members had the full syndrome; however, patients' relatives did not undergo muscle biopsy or molecular studies.

Increased lactate levels in the serum, and particularly in the CSF, are important for the diagnosis of MELAS, because it may indicate mitochondrial dysfunction in al-

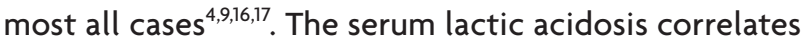
with the severity of neurological impairment, and CSF lactate level may be particularly elevated during the strokelike episodes ${ }^{16,17}$. Other clinical investigations for MELAS such CK, AL and EMG (myopathic pattern) were less specific in our study.

Cardiac involvement is described up to half of the patients with this disease. It includes preexcitation, atrioventricular heart block, rhythm abnormalities and dilated or hypertrophic cardiomyopathy (particularly left ventricular hypertrophy), but is not the predominant symptom; it is not known whether isolated subtle abnormalities progress in time to major cardiac abnormalities ${ }^{3,4,18,19}$. However, all patients with MELAS should undergo routine cardiac examinations because the described cardiac abnormalities may have important therapeutic and prognostic consequences $^{18}$.

Brain CT or MRI studies in MELAS show cortical and white matter lesions (stroke-like lesions) of varying size and side in the temporo-parieto-occipital region, basal ganglia, brainstem, or cerebellum ${ }^{3,14,16,20}$. On cerebral MRI the acute stroke-like lesions start from a focal temporal lesion that, in one-third of the patients, moves to the surrounding parietal or occipital areas ${ }^{20}$. Furthermore, some brain imaging studies reveal predominance of stroke-like lesions in one or both occipital lobes, but the reason for this tendency is unclear ${ }^{9,21}$. A T1-weighted hyperintense cortical signal on MRI compatible with cortical laminar necrosis can be seen during the subacute stage of stroke-like lesions ${ }^{20}$. The distribution pattern of these lesions does not correspond to any vascular territory ${ }^{4,4,1,6,21}$. Later calcification may be seen in the basal ganglia and diffuse cerebral or cerebellar atrophy can occur ${ }^{3,416,21}$. The spreading mechanisms of stroke-like lesions also remain unknown, but may be due to vascular abnormalities resulting from mitochondrial angiopathy or a metabolic disorder caused by mitochondrial dysfunction-induced energy failure ${ }^{5,7,9,14,16,221}$.

The RRF is the histological hallmark of proliferation of abnormal mitochondria in muscle fibers ${ }^{1,22,23}$. Identification of any RRF in a young person ( $<30$ years old) is suspicious of mitochondrial disease and levels above $2 \%$ can be regarded as a diagnostic indicator at any age ${ }^{1,23}$. In children with mitochondrial diseases, true RRF are a rare finding but subsarcolemmal accumulations of mitochondria are much more common, and often increase with the age until they finally comprise true $\mathrm{RRF}^{23}$. Thus, recognition of subsarcolemmal accumulation of mitochondria without typical RRF in younger patients can be considered one minor histology criteria for mitochondrial disease diagno$\mathrm{sis}^{23}$. The accumulation of mitochondria in muscle fibers composing typical RRF has been found in up to $97 \%$ of MELAS patients ${ }^{4,7,15}$. In our group, more than $2 \%$ RRF were found in almost all patients but, as in other mitochondrial diseases, the SDH was a more sensitive marker of mitochondrial proliferation than the usual MGT stains ${ }^{13}$. The incidence of RRF on MGT and SDH stain in MELAS A3243G patients was similar to our other MELAS patients.

Importantly, two frequent morphologic abnormalities found on muscle biopsy distinguish MELAS from other mitochondrial diseases: the large proportion of RRF with normal COX activity and the presence of SSV ${ }^{3,24}$.

The COX activity can be diffusely decreased or focally absent in some muscle fibers, but in these cases the COX probably is not the primary defective site of disease $e^{9,15,22}$. Thus, most of the MELAS patients have normal or increased COX activity, as in this study, possibly because the mtDNA mutation is insufficient to impair COX activity ${ }^{22,25}$. In our patient with COX deficiency, the number of COXdeficient fibers was greater than RRF frequency, possibly due to the greater number of muscle fiber with mitochondrial dysfunction. In addition, COX activity is usually normal in most of the RRF and reactive blood vessels, as in the present study ${ }^{7,26,27}$.

SSV are characteristically found in mitochondrial encephalomyopathies, including MELAS, and can affect brain and intestinal vessels as well as skeletal muscle vessels ${ }^{7,22,24,28}$. This focal and segmental proliferation of vascular mitochondria in MELAS is thought to be a compensatory reaction to the respiratory chain dysfunction, which has been well documented at autopsy by histochemical and immunohistochemical studies ${ }^{24}$. This vascular involvement in patients with MELAS has been studied by several investigators, but the pathogenic significance of these findings is unknown; however, some investigators have speculated that blood vessel changes indicate that MELAS is a generalized angiopathy ${ }^{3}$.

The histological studies of MELAS patients, whatever their genetic background, has demonstrated a high SSV incidence (almost $90 \%)^{7,15,28}$. SSV have previously been found to be associated with MELAS phenotype in Brazilian patients, but our study found a lower SSV incidence (60\%) than other studies ${ }^{7,915}$. In this study, the patients without SSV are younger and the evolution of their disease shorter compared with SSV patients studied by Hasegawa et al. ${ }^{28}$. We believe that SSV formation is similar to the RRF formation, which varies according to the time when the biopsy was performed and the severity of the disease. Thus, the blood vessels can be normal in early age but show SSV on $\mathrm{SDH}$ stain in patients at late stages of the disease. 
The presence of SSV in muscle biopsy specimens provides a relevant feature that can help to confirm the MELAS diagnosis, especially in patients without RRF in their muscle biopsy, but the lower incidence of SSV in our patients suggests that its absence should not be used as an exclusion criterion for MELAS $S^{7,15,28}$.

The degree of heteroplasmia (the proportion of normal and mutant mtDNA in each tissue) is an important factor influencing variability of phenotypical presentation ${ }^{9,15,22}$. Muscle biopsies from most MELAS cases, especially those associated with the most common mutation, A3243G in the tRNA ${ }^{\text {Leu(UUR) }}$ gene, have RRF with normal COX activity and SSV ${ }^{24}$. Quantitative analysis showed 80 to $90 \%$ mutant mtDNA in muscle ${ }^{25-27}$. The total mtDNA (both normal and mutant) are extremely increased in SSV and RRF ${ }^{25-27}$. The proportion of mutant mtDNA was significantly higher in RRF than in non-RRF, as well as in SSV than in non-SSV cases $^{26,27}$. The similar morphological properties of these fibers and vessels suggest that increased mutant mtDNA is responsible for dysfunction and mitochondrial proliferation in both tissues of MELAS patients where COX is not a primarily defective enzyme ${ }^{25-27}$.

The present study suggested an association between SSV and A3243G mutation because all patients with this mutation had SSV. However, this study identified few cases with A3243G mutation.

The proportion of mutated mtDNA in blood cells is lower than in muscle (heteroplasmia), because of the turnover of mitochondria in this tissue ${ }^{23}$. Therefore, the muscle was used in this study because genetic analysis of blood cells is not absolutely reliable as a diagnostic test and a negative result does not exclude mtDNA mutation ${ }^{23}$.

Only 6 years after its first clinical description, a point mutation in the mIDNA was associated with the MELAS syndrome ${ }^{29}$. A3243G mutation is the most frequent of the mtDNA mutations and causes a wide range of clinical disease, of which MELAS is the most prevalent ${ }^{5,14}$. A3243G mutation occurs in approximately $80 \%$ of MELAS patients, but the present study showed association of $A 3243 \mathrm{G}$ mutation with MELAS in $57.1 \%$ of patients, possibly because this study had few cases for molecular analysis ${ }^{3,5,713,15}$. Due to the high frequency of this mutation, PCR/RLFP for the A3243G mutation must be the first molecular test when MELAS is suspected and is a simple molecular test for this genetic defect. The A3243G mutation accounts in approximately $80 \%$ of MELAS patients, but it is not the only genetic defect reported. Other point mutations in the same gene can be associated with MELAS $S^{9,13,15}$. The second most common mtDNA point mutation in MELAS patients is T3271C, which occurs in $7.5 \%$. Brazilian patients were previously reported with this mutation, but we did not find this mutation in our group'. We suggest that the second genetic diagnostic test should be the PCR/RLFP for T3271C ${ }^{7,9,13}$. MELAS could be also caused by mtDNA point mutations in other genes, as the tRNA ${ }^{\text {phe }}$, tRNA ${ }^{\text {Val }}$, tRNA ${ }^{\text {Lys }}$, COXIII, NDI, ND5 or rRNA, or by small-scale mtDNA deletions $s^{2,7}$. However, as the tRNA ${ }^{\text {Leu(UUR) }}$ mutations are still the most commonly involved in the MELAS phenotype, we also speculate that molecular analysis of this gene by direct sequencing should be the third molecular test in MELAS patients ${ }^{7,24}$.

In our patients, the common mutation A3243G was found in 4 cases. Therefore, in MELAS with atypical biochemical or histological findings, such as COX deficiency, the putative mtDNA mutation might not involve one of the common tRNA ${ }^{\text {Leu(UUR) }}$ mutations ${ }^{24}$. In these cases, after sequencing the tRNA ${ }^{\text {Leu(UUR) }}$ gene, the other possible "hot spots" for mtDNA mutation in MELAS were in the tRNA ${ }^{\mathrm{val}}$, tRNA ${ }^{\text {Lys }}$, ND1 or ND5 genes (all of which might be sequenced for diagnostic purpoes) $)^{3,5,14,24}$.

Our study reveals that muscle biopsy often confirms the MELAS diagnosis by presence of RRF on MGT and SDH stain, and SSV in muscle biopsy specimens. Molecular analysis of tRNA ${ }^{\text {Leu(UUR) }}$ gene should not be the only diagnostic criteria for MELAS.

\section{REFERENCES}

1. Walker UA, Collins S, Byrne E. Respiratory chain encephalomyopathies: a diagnostic classification. Eur Neurol 1996;36:260-267.

2. Schmiedel J, Jackson S, Schafer J, Reichmann H. Mitochondrial cytopathies. J Neurol 2003;250:267-277.

3. Hirano M, Pavlakis SG. Mitochondrial myopathy, encephalopathy, lactic acidosis, and stroke-like episodes (MELAS): current concepts. J Child Neurol 1994;9:4-13.

4. Hirano M, Ricci E, Koenigsberger MR, et al. MELAS: an original case and clinical criteria for diagnosis. Neuromuscul Disord 1992;2:125-135.

5. Zeviani M, Di Donato S. Mitochondrial disorders. Brain 2004;127:21532172.

6. Pavlakis SG, Phillips PC, DiMauro S, DeVivo DC, Rowland LP. Mitochondrial myopathy, encephalopathy, lactic acidosis, and stroke-like episodes: a distinctive clinical syndrome. Ann Neurol 1984;16:481-488.

7. Goto Y. Clinical features of MELAS and mitochondrial DNA mutations. Muscle Nerve 1995;20(Suppl 3):S107-S112.

8. Werneck LC, Abdalla H, Lohr A. MELAS (mitochondrial encephalopathy, lactic acidosis and stroke like episodes): case report. Arq Neuropsiquiatr 1987;45:288-294.

9. Marie SKN, Goto Y, Passos-Bueno MR, et al. A Caucasian family with the 3271 mutation in mitochondrial DNA. Biochem Med Metab Biol 1994;52:136-139.

10. Conforto AB, Yamamoto FI, Oba-Shinjo SM, et al. Screening for MELAS mutations in young patients with stroke of undetermined origin. Arq Neuropsiquiatr 2007;65:371-376.

11. Werneck LC. The value of muscle biopsy in neurology: a study of 290 biopsies. Rev Bras Clin Ter 1981;10(Suppl):S2-S24.

12. Anderson S, Bankier AT, Barrell BG, et al. Sequence and organization of the human mitochondrial genome. Nature 1981;290:457-465.

13. Ciafaloni E, Ricci E, Shanske S, et al. MELAS: clinical features, biochemistry, and molecular genetics. Ann Neurol 1992;31:391-398.

14. Finsterer J. Genetic, pathogenetic, and phenotypic implications of the mitochondrial A3243G tRNALeu(UUR) mutation. Acta Neurol Scand 2007;116:1-14.

15. Goto Y, Horai S, Matsuoka T, et al. Mitochondrial myopathy, encephalopathy, lactic acidosis, and stroke-like episodes (MELAS): a correlative study of the clinical features and mitochondrial DNA mutation. Neurology 1992;42:545-550. 
16. Finsterer J. Central nervous system manifestations of mitochondrial disorders. Acta Neurol Scand 2006;114:217-238.

17. Kaufmann P, Shungu DC, Sano MC, et al. Cerebral lactic acidosis correlates with neurological impairment in MELAS. Neurology 2004;62: 1297-1302.

18. Vydt TGC, Coo RFM, Soliman OI, et al. Cardiac involvement in adults with m.3243A>G MELAS gene mutation. Am J Cardiol 2007;99:264-269.

19. Okajima $\mathrm{Y}$, Tanabe $\mathrm{Y}$, Takayanagi M, Aotsuka H. A follow up study of myocardial involvement in patients with mitochondrial encephalomyopathy, lactic acidosis, and stroke-like episodes (MELAS). Heart 1998;80: 292-295.

20. lizuka T, Sakai F, Kan S, Suzuki N. Slowly progressive spread of the stroke-like lesions in MELAS. Neurology 2003;61:1238-1244.

21. Haas R, Dietrich R. Neuroimaging of mitochondrial disorders. Mitochondrion 2004;4:471-490.

22. Bourgeois JM, Tarnopolsky MA. Pathology of skeletal muscle in mitochondrial disorders. Mitochondrion 2004;4:441-452.

23. Bernier FP, Boneh A, Dennett X, Chow CH, Cleary MA, Thorburn DR. Diagnostic criteria for respiratory chain disorders in adults and children. Neurology 2002;59:1406-1411.
24. Tanji K, Kaufmann P, Naini AB, et al. A novel tRNAVal mitochondrial mutation causing MELAS. J Neurol Sci 2008;270:23-27.

25. Petruzzella V, Moraes CT, Sano MC, Bonilla E, DiMauro S, Schon EA Extremely high levels of mutant mtDNA co-localize with cytochrome c oxidase-negative ragged-red fibers in patients harboring a point mutation at nt 3243. Hum Mol Genet 1994;3:449-454.

26. Tokunaga M, Mita S, Sakuta R, Nonaka I, Araki S. Increased mitochondrial DNA in blood vessels and ragged-red fibers in mitochondrial myopathy, encephalopathy, lactic acidosis, and stroke-like episodes (MELAS). Ann Neurol 1993;33:275-280.

27. Tokunaga M, Mita S, Murakami T, et al. Single muscle fiber analysis of mitochondrial myopathy, encephalopathy, lactic acidosis, and strokelike episodes (MELAS). Ann Neurol 1994;35:413-419.

28. Hasegawa H, Matsuoka T, Goto Y, Nonaka I. Strongly succinate dehydrogenase-reactive blood vessels in muscle from patients with mitochondrial myopathy, encephalopathy, lactic acidosis, and stroke-like episodes. Ann Neurol 1991;29:601-605.

29. Goto Y-I, Nonaka I, Horai S. A mutation in the tRNA ${ }^{\text {Leu(UUR) }}$ gene is associated with the MELAS subgroup of mitochondrial encephalomyopaties. Nature 1990;348:651-653. 